\title{
Identification of Diabetic Retinopathy Stages using Fuzzy C-means Classifier
}

\author{
Shahira M. Habashy \\ Electronic and Communication Department, Engineering Collage, Helwan University, Cairo, Egypt
}

\begin{abstract}
Diabetic Retinopathy (DR) is globally the primary cause of visual impairment and blindness in diabetic patients. Diabetic retinopathy occurs when the small blood vessels have a high level of glucose in the retina. That causes a change in the retina, which occur over a period of time in diabetics and cause the difficulties with vision. Regular screening is essential in order to detect the early stages of diabetic retinopathy for timely treatment to prevent or delay further deterioration. In this paper, the presences of abnormalities in the retina such as the structure of blood vessels, microaneurysms, and exudates using image processing techniques are detected. These features are processed with the help of Fuzzy C-Means clustering algorithm to detect the different diabetic retinopathy stages. This system intends to help ophthalmologists in DR screening process to detect symptoms faster and more easily. The sensitivity, Precision and accuracy for that Diabetic Retinopathy detection system are $98.01 \%, 99 \%$, and $97 \%$ respectively.
\end{abstract}

\section{Keywords}

Diabetic retinopathy (DR), microaneurysms, exudates and Fuzzy C-Means (FCM), Retinal Image (RI).

\section{INTRODUCTION}

Diabetic retinopathy is the name given to the changes in the retina, which occur over a period of time in diabetics. The retina is the back part of the eye and is made up of cells, which are sensitive to light. It is fed by a network of blood vessels and any change in these blood vessels can cause the difficulties with vision. The walls of the blood vessels become fragile and then start to break, leaking blood around them. Sometimes, before the walls actually break, the weakened area can be seen, by the person who examines the eye, to have ballooned out. These are called micro-aneurysms. If these break, the amount of blood which leaks out is fairly small, and the only symptoms may be a few areas of blurring or floating spots in front of the eyes [1]. Later the blood vessels may stop carrying blood permanently, and the cells in the retina will die from lack of nourishment. This kind of loss of sight is gradual but at the present time, it is permanent. When old blood vessels close down, new but abnormal ones will grow to take their place. They are unable to nourish the retina properly, and may grow into the transparent inner part of the eye, and further affect vision [2]. Retinal images obtained by the fundus camera are used to diagnose DR. Automated methods of DR screening help to save time, cost and vision of patients, compared to the manual methods of diagnosis [3-4]. The screening of diabetic patients for the development of diabetic retinopathy can potentially reduce the risk of blindness in these patients by $50 \%$. An early detection enables laser therapy to be performed to prevent or delay visual loss and may be used to encourage improvement in diabetic control [56]. Generally, diabetic retinopathy is classified into two main stages, namely nonproliferative diabetes retinopathy (NPDR) and proliferative diabetes retinopathy (PDR). nonproliferative diabetes retinopathy, can be classified to mild, moderate or severe. This classification are depending on the presence and extent of the features such as hard exudates, microaneurysms or cotton wools spots due to leakage of fluid and blood from the blood vessels Mild NPDR is the earliest stage of retinopathy and vision is usually normal except in some cases. However, deterioration of the blood vessels in the retina has already started. Blood vessels erupt when there is not enough oxygen in the blood because of high levels of glucose microaneurysms may occur. These manifestations of the disease are small areas of balloon-like swelling in tiny blood vessels [7]. Moderate NPDR occur when the disease progresses. Some of the blood vessels that irrigate the retina become blocked. It is more than "mild" but less than "severe" stage. There will be micro-aneurysms or hemorrhages of greater severity in one to three quadrants and leakage might occur, resulting cotton wool spots and exudates etc to be present in the retina. The dangers stage (Severe NPDR) happened as more blood vessels are blocked, those areas in the retina will be deprived of blood supply. Signals will then be sent to the body for the growth of new vessels in order to compensate for the lack of nourishment [7-8]. In Proliferative Retinopathy, more signals sent by the retina for nourishment trigger the growth of new blood vessels. These new blood vessels are abnormal and fragile. They grow along the retina and along the surface of the clear, vitreous gel that fills the inside of the eye. By themselves, these blood vessels do not cause symptoms or vision loss. However, they have thin, fragile walls. If they leak blood, severe vision loss and even blindness can result [9]. Fig. 1 shows fundus image

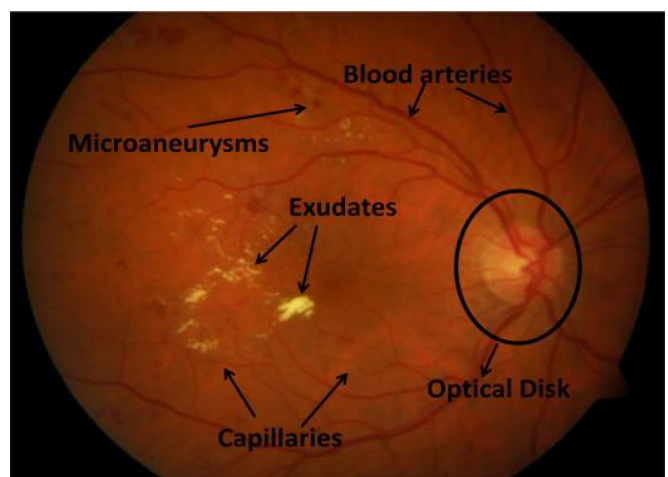

Figure 1, Fundus Image

Detection of these visual elements remains difficult for several reasons. First, hemorrhages and microaneurysms are 
often difficult to distinguish from blood vessels, even for a trained professional. Larger hemorrhages are of the same color as blood vessels but typically differ geometrically. However, smaller hemorrhages and microaneurysms are often similar in color, geometry and texture to the thin root-like components of the blood vessel network. In a similar vein, exudates and optic discs share similar color spaces. For this reason, many previous attempts have first tried to localize and mask the blood vessel network and the optic disc. However, many images are clouded by non-retinopathic features making feature extraction so difficult. Finally, the color variation among exudates and hemorrhages in a single image is far less than the overall variation among these retinopathy components across all images [7]. Many searches were detected diabetic retinopathy. In [10] color features on a Bayesian statistical classifier used to classify each pixel into lesion or non-lesion classes. In [11] a thresholding technique based on the selection of regions to detect exudates was applied. A patch of size $256 \times 192$ pixels is selected over the area of interest. Large exudates were detected using Global thresholding, while local thresholding is used to detect the lower intensity exudates. An automatic detection of diabetic retinopathy using an artificial neural network was proposed in [12]. The exudates are identified from grey level images and the fundus image is analyzed using a back propagation neural network. The classification of a $20 \times 20$ region is used rather than a pixel-level classification. The Fuzzy C-Means (FCM) clustering is a well-known clustering technique for image segmentation. It was developed by Dunn [13] and improved by Bezdek [14]. In [15] color normalization and a local contrast enhancement in a pre-processing step. The color retinal images are segmented using Fuzzy C-Means (FCM) clustering and the segmented regions are classified into two disjoint classes - exudate and nonexudate patches - using a neural network. The comparative exudate classification using Support Vector Machines (SVM) and neural networks was also applied. They showed that SVM are more practical than the other approaches [16]. Other Retinal image classification techniques have been done. In [17] MDD classifiers for classifying retinal images are used. Propagation through radii method is used for feature extraction. a computer based approach for the detection of diabetic retinopathy stage using SVM is performed in [18]. The features are extracted from the raw images using image processing techniques. Retinal Grading Algorithm was used by [19] to automatically classify the DR intensity based on distribution of exudates, count, size and the distribution of the hemorrhages and microaneurysms. Classification using fractal measures and clustering techniques was done by [20]. Multiple classifiers were used by [21] for the classification of retinal images. In this work, segmentation is done on retinal images to separate the blood vessels, exudates and microaneurysms (MA) since these are the best indicators of the presence of DR. The features given to the classifier include the areas of these segmented structures. Fuzzy c-mean classifier classifies the input image as normal (not affected by DR), Mild, Higher, and serve stages to classify the NPDR and PDR. This classification is based on the training done by giving the sample features. Database used consist of DIARETDB1 [22] and DIARETDB0 [23]. DIARETDB1 database consists of 89 color fundus images of which 84 contain non-proliferative signs of the diabetic retinopathy, and 5 are normal which do not contain any signs of the diabetic retinopathy. DIARETDB0 database consists of 130 colors fundus images of which 20 are normal and 110 contain signs of the diabetic retinopathy (hard exudates, soft exudates, micronaneuyrysms, hemorrhages and neovascularization). Images were captured with a 50 degree field-of-view digital fundus camera with unknown camera settings. The data correspond to practical situations, and can be used to evaluate the general performance of diagnosis methods. This data set is referred to as "calibration level 0 fundus images". The rest of the paper is organized as follows. Section 2 provides a more overview of the Fuzzy C-mean classifier. Section 3 explains fundus images Methodology features extraction. Section 4 reviews classification results. Conclusion explains in Section 5.

\section{SEGMENTATION USING FUZZY C- MEANS CLUSTRING}

Segmentation using Fuzzy C-Means (FCM) Clustering is an overlapping clustering algorithm, where each point may belong to two or more clusters with different degrees of membership. The similarity is defined by the distance of the features vector to the cluster centers. Euclidean distance is used to measure this distance and data will be associated to an appropriate membership value [24-25]. The cluster center is updated until the difference between adjacent objective function, as displayed in eq. 1 is close to zero or practically less than a predefined small constant.

$$
\begin{aligned}
& J=\sum_{i=1}^{N} \sum_{j=1}^{C} U_{i j}^{2}\left\|X_{i}-C_{j}\right\|^{2} \\
& U_{i j}=\frac{1}{\sum_{K=1}^{C}\left(\frac{\left\|X_{i}-C_{j}\right\|}{\left\|X_{j}-C_{k}\right\|}\right)^{2}} \\
& C_{j}=\frac{\sum_{i=1}^{N} U_{i j}{ }^{2} X_{i}}{\sum_{i=1}^{N} U_{i j}{ }^{2}}
\end{aligned}
$$

where $N$ is number of features, 3 for our case. $C$ is number of clusters, which take as 4 in this search. $u_{i j}$ is the degree of membership of $x_{i}$ in the cluster $j, x_{i}$ is the $i$ th of d-dimensional measured data, $c_{j}$ is the d-dimension center of the cluster, and $\|*\|$ is any norm expressing the similarity between any measured data and the center. The update of membership $u_{i j}$ and the cluster centers $c_{j}$ are carried by eq. 2 and eq. 3 , respectively. This iteration will stop when Eq. (4) is satisfied.

$\max _{i j}=\left\{\left|U_{i j}{ }^{K+1}-U_{i j}{ }^{K}\right|\right\}<\varepsilon$

Where $\varepsilon$ is a termination criterion, 0.00001 for our case. $k$ is the maximum iteration step, 200 for our case.

\section{METHODOLOGY}

Three different features from the fundus images namely Blood vessels, Exudates, and Microaneurysms are extracted using image processing techniques. Those values are essential as they represent the image and are necessary in order to classify the images accurately. Fuzzy C-means Clustering is used to classify the input fundus images into four classes (Normal, Mild, Moderate and Severe).

\subsection{Preprocessing}

Firstly, original image's Red, Green and Blue (RGB) space was transformed to grayscale. The contrast of the fundus images tends to be bright in the centre and diminish at the side, hence Adaptive Histogram Equalization is essential to minimize this effect and have a more uniform image. Image 
segmentation is then performed to adjust the contrast intensity and small pixels considered to be noise are removed with the aid of median filter. Original intensity band image and intensity bands after preprocessing are shown in Fig. 2 (a) and Fig. 2 (b), respectively.

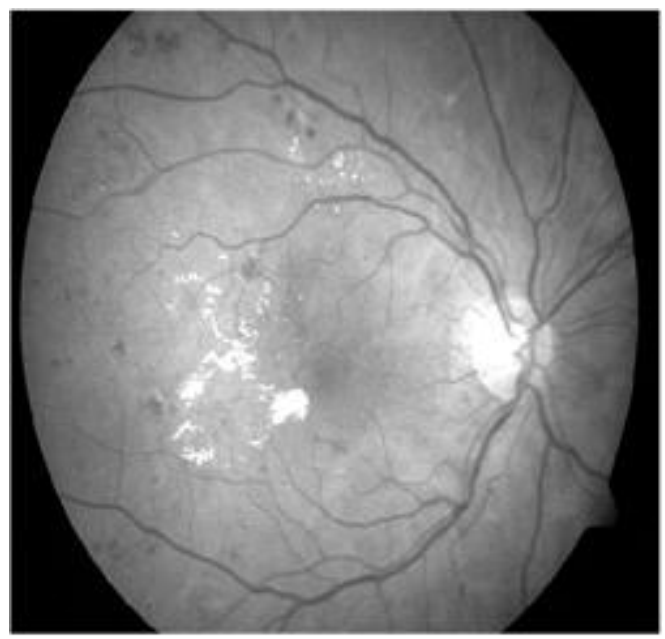

Fig. 2a, the original image

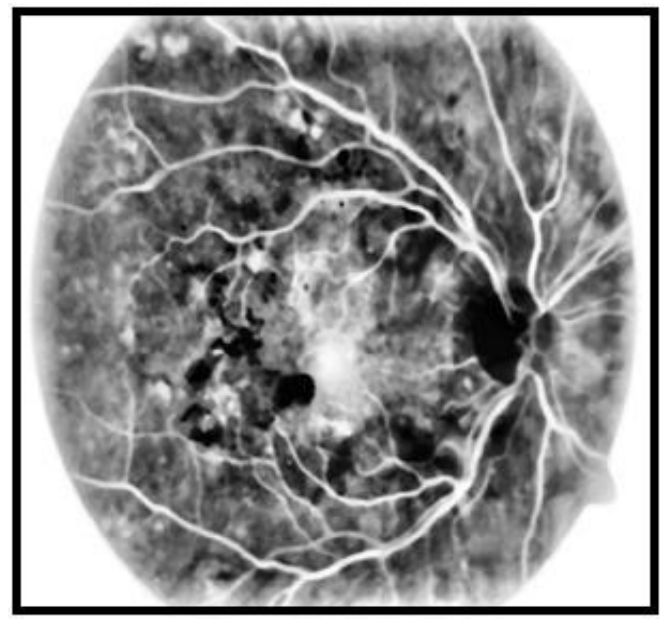

Fig. $2 b$, the preprocessing image

\subsection{Segmentation of Retinal Structures}

\section{3-2-1 -Blood Vessels Detection}

Blood vessels shape and size are considered as indication to exist of diabetic retinopathy and its degree. The higher stage of the diabetic retinopathy is known to have more blood vessels due to eye damages. Morphological opening which consisted of erode followed by dilate is applied first. Erode function protects the small blood vessels by reducing their sizes while dilate function blows up the larger remaining details which are intended to be removed. Second the resulted image was subtracted from the preprocessing image. The resulted image is shown in fig. 3 after converting it to binary and removing the small area of pixels considered as noise.
Area of blood vessels is determined by finding the total number of white (vessel) pixels in the vessel-segmented image.

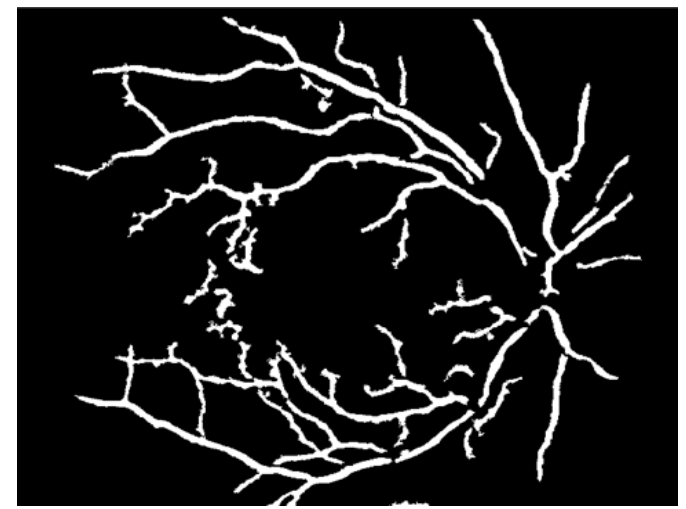

Fig. 3, the Blood Vessels Detection

\section{3-3-2 Exudates Detection}

The exudates can be identified on the ophthalmoscope as areas with hard white or yellowish colors and varying sizes, shapes and locations, near the leaking capillaries within the retina. Main cause of exudates is proteins and lipids leaking from the blood into the retina via damaged blood vessels. Morphological dilate is applied to the preprocessing image. The dilate function expands the exudates area. This image is converted to binary with a threshold value to filter out the bright regions fig.4a. The grayscale image and preprocessing image are also converted to binary with different thresholding values to extract the bright spots. Fig. $4 \mathrm{~b}$ shows the binary representation of grayscale image. By applying AND logic to those three binary images, the exudates and optical disk areas are obtained as a result fig. 4c. At this stage the optic disk area need to be removed. The procedure applied to remove optical disk is as in [26]. Finally the exudates area is obtained as in fig. $4 \mathrm{~d}$. Area of exudates is determined by finding the number of white pixels in the exudates image.

\section{3-3-3 Microaneurysms Detection}

Microaneurysms appeared as small dark round dots ( 15 to 60 microns in diameter) on the fundus images. They are small bulges developed from the weak blood vessels and are the earliest clinical sign of diabetic retinopathy. The number of microaneurysms would increase with the stage of the retinopathy. For detecting Microaneurysms, the preprocessing image first converted to binary image. Second find image edges using canny edge detection as shown in fig. 5a. Then the program filled the holes found from the last stage. Fig. 5b shows that, not all the holes filled represented Microaneurysms but also some Blood Vessels are filled. Fig. $5 \mathrm{c}$ shows the result image after subtracting fig. $5 \mathrm{~b}$ from the preprocessing image. This image is compared using AND logic with the result from the Blood Vessels detection to select the holes result from Blood Vessels. Then subtract fig. $5 \mathrm{~d}$ from fig. $5 \mathrm{c}$ to detect Microaneurysms only as shown in fig.5e. Area of Microaneurysms is determined by finding the number of white pixels in the result image. 


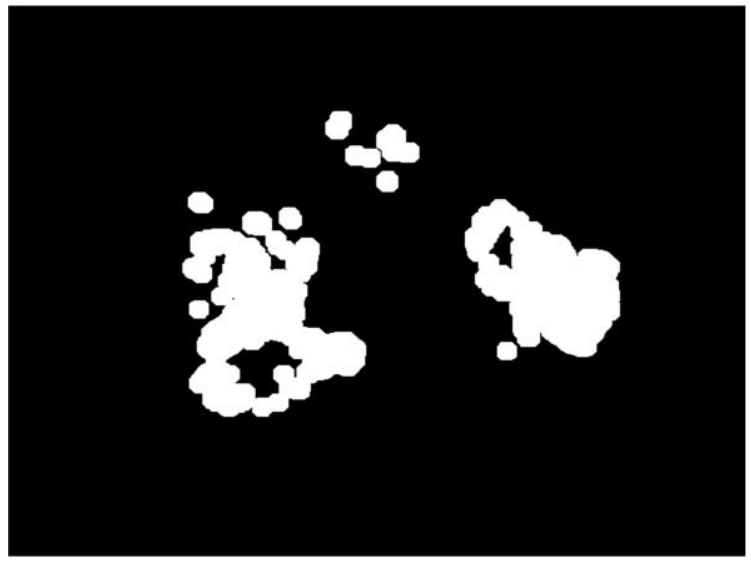

(a)

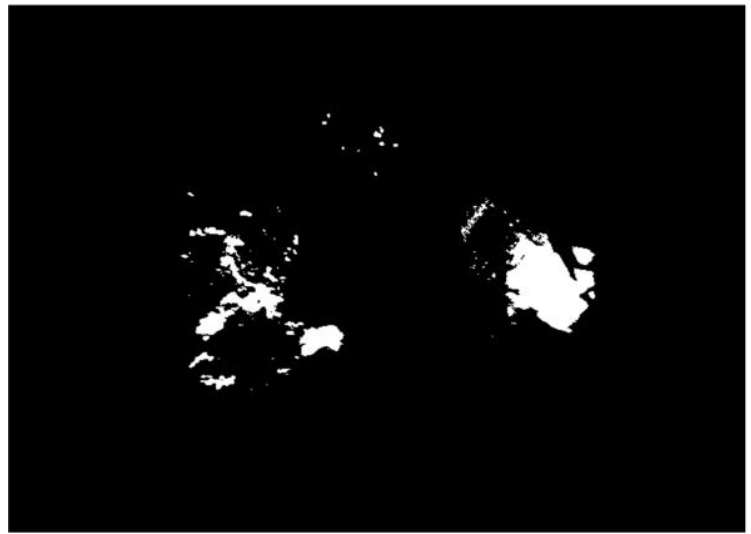

(c)

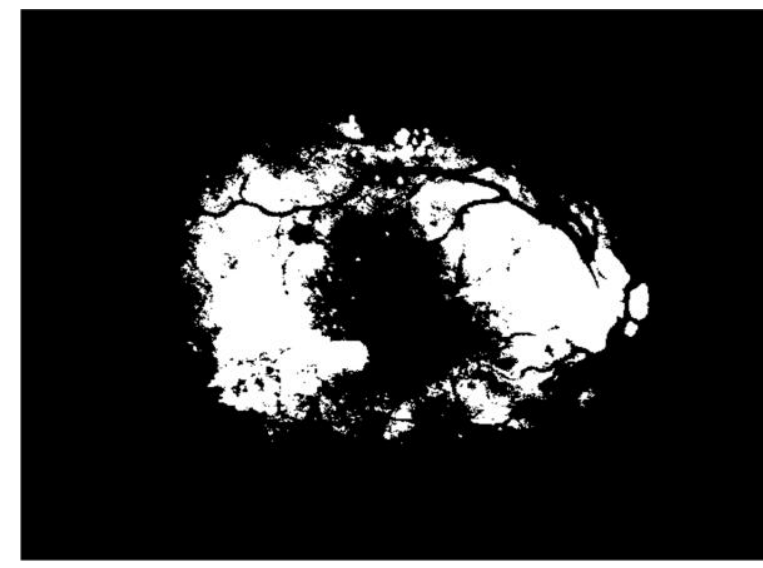

(b)

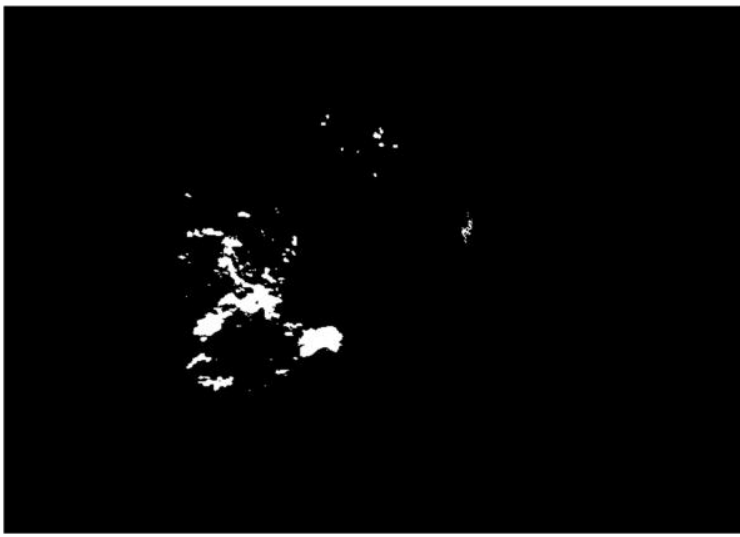

(d)

Fig. 4 Exudates segmentation (a) binary representation of applying the dilate function on the preprocessing image (b) binary representation of grayscale image (c) exudates and optical disk areas (d) Exudates result area

\section{4- RESULTS}

The proposed algorithm is implemented in MATLAB environment. The proposed system was constructed and tested using DIARETDB1 [22] and DIARETDB0 [23]. Those databases are divided into training and evaluation dataset to assess the performance of the proposed method. Training database (100 fundus images) objectives to find the classes' center. First, fundus images are pre-processed in order to improve image contract. Second, Image segmentation is applied in order to extract three features namely Blood vessels, Exudates, and Microaneurysms. Those features are among the preliminary signs of diabetic retinopathy, a major cause of vision loss in diabetic patients. Early detection of diabetic retinopathy could improve patients' chance to avoid blindness. Those features' values are then fed into fuzzy cmeans classifier for classification to four classes (Normal, Mild, Moderate or Severe). This classification was performed according to the number of pixels segmented as Blood vessels, Exudates, and Microaneurysms respectively. This stage output was the four centers related to the four diabetic retinopathy classes. Fig. 6, shows progress in determining values of the objective function during fuzzy c-mean iteration. The second part of Database (100 fundus images) is used for the proposed system evaluation. The same processes are applied on those fundus images to extract Blood vessels,
Exudates, and Microaneurysms feature values. In this stage, a method is needed to classify images based on the extracted feature vectors. The minimum Euclidean distance related to the four center determined from the last stage is used for that classification. To evaluate the medical diagnosis systems, Sensitivity and Precision criteria are usually used. eqs. 5 and 6 define these two criteria [27-28].

Sensivity $=\frac{\mathrm{TP}}{\mathrm{TP}+\mathrm{FN}}$

Precision $=\frac{\mathrm{TP}}{\mathrm{TP}+\mathrm{FP}}$

In these equations, TP, FN and FP show the number of true positive, number of false negative and number of false positive samples respectively. In classification systems, accuracy is often used to evaluate the systems. Accuracy is the overall correctness of the system and is calculated as the sum of correct classifications divided by the total number of classifications as in eq. 7.

Accuracy $=\frac{\text { items calssified correctly }}{\text { all items classified }}$ 
It is found that the proposed method detects classes successfully with sensitivity, Precision, and accuracy of

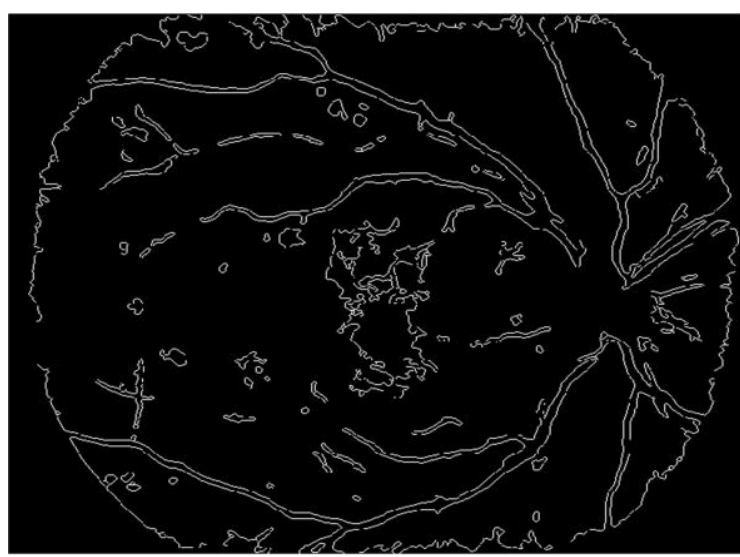

(a)

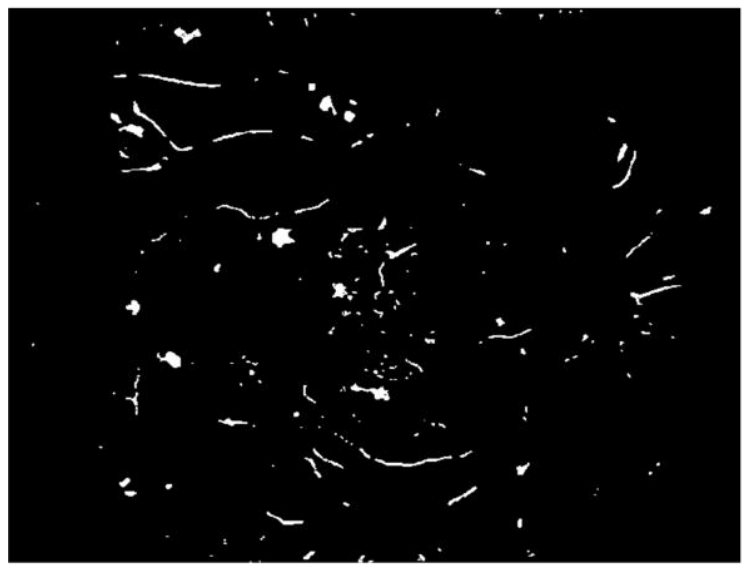

(c)
$98.01 \%, 99 \%$, and $97 \%$ respectively.

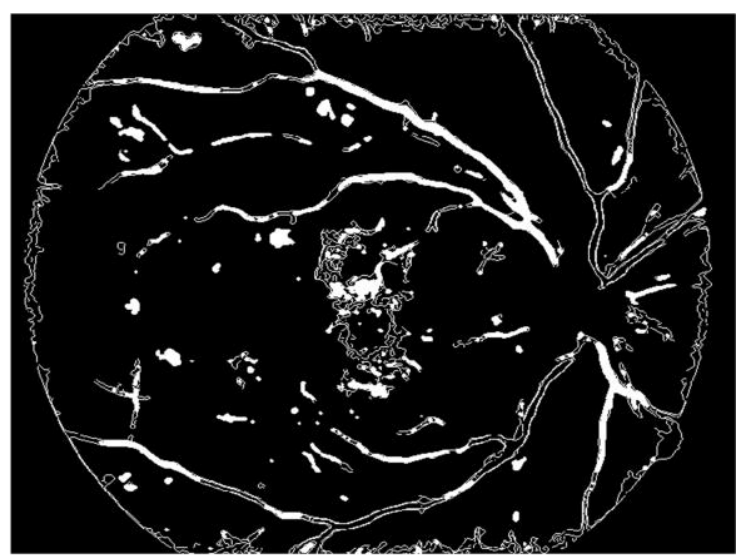

(b)

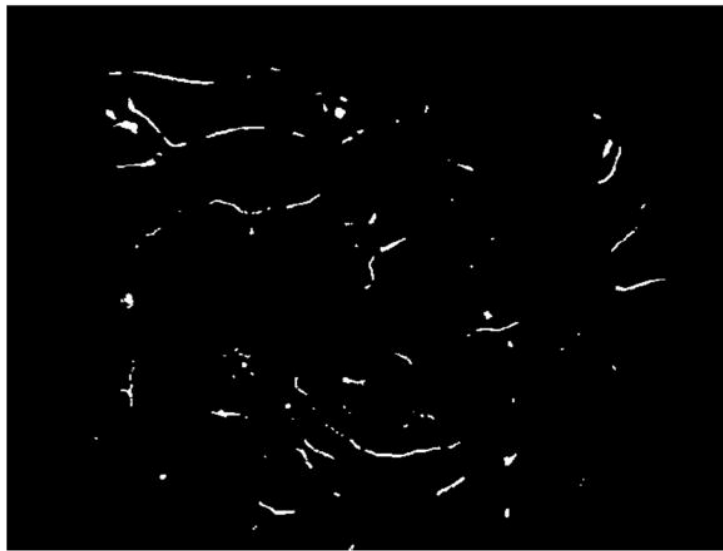

(d)

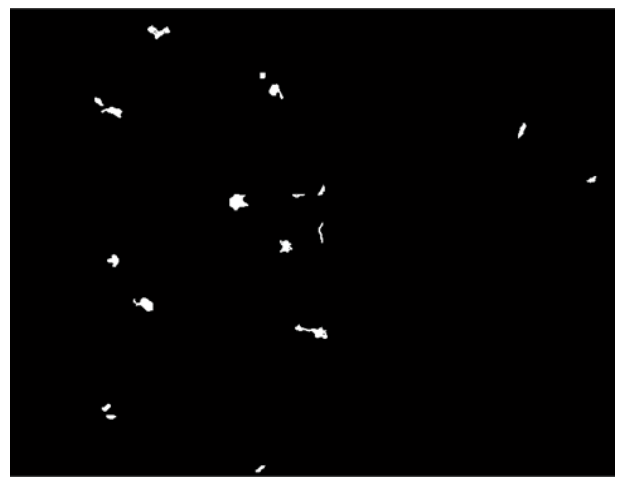

(e)

Fig. 5 Microaneurysms Segmentation (a) canny edge detection (b) canny edge image after filled holes (c) result image after subtracting fig. $5 \mathrm{~b}$ from the preprocessing image. (d) result of comparing fig.5-c with fig. 3. (e) Microaneurysm image 


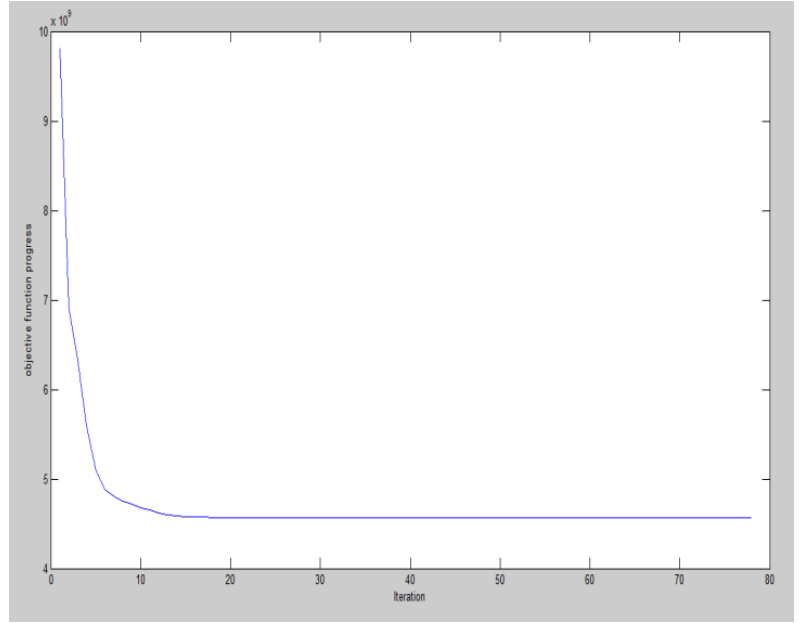

Fig. 6, progress in determining values of the objective function during fuzzy c-mean iteration.

\section{CONCLUSIONS}

Diabetic retinopathy eye diseases are the main cause of vision loss and their prevalence is set to continue rising. Current methods of detection and assessment of diabetic retinopathy are manual, expensive and require trained ophthalmologists. Automatic Diabetic retinopathy eye diseases detection would be helpful for diabetic retinopathy screening Process. Early detection can potentially reduce the risk of blindness. An automatic method to detect Diabetic retinopathy eye diseases using a Fuzzy C-Means (FCM) clustering is proposed. There are two modules in this work, one that performs image segmentation which includes the segmentation of vessels, exudates and MA and other one that performs classification using fuzzy c-mean classifying. Contrast enhancement preprocessing is applied before three features, namely blood vessels, exudates and MA are extracted to supply as input parameters to coarse segmentation using FCM clustering method. Sensitivity, Precision, and accuracy are used to evaluate overall performance. It is found that the proposed method detects Diabetic retinopathy eye class successfully with sensitivity, Precision, and accuracy of $98.01 \%, 99 \%$, and 97\% respectively.

\section{REFERENCES}

[1] A. Osareh, M. Mirmehdi, B. Thomas and R. Markham. 2003 Automated Identification of Diabetic Retinal Exudates in Digital Colour Images. British Journal of Ophthalmology, 87(10), 1220-1223.

[2] C. Sinthanayothin, J.F. Boyce, H.L. Cook, T.H. 1999 Williamson, Automated Localization of the Optic Disc, Fovea, and Retinal Blood Vessels from Digital Colour Fundus Images. British Journal of Ophthalmology, 83, 231-238.

[3] C.I. Sanchez, R. Hornero, M.I. Lopez, J. Poza. 2004 Retinal Image Analysis to Detect and Quantify Lesions Associated with Diabetic Retinopathy. Proc. 26 IEEE Annual International Conf. on Engineering in Medicine and Biology Society (EMBC), 3, $1624-1627$.

[4] Selvathi D, N.B.Prakash, and Neethi Balagopal. 2012 Automated Detection of Diabetic Retinopathy for Early
Diagnosis using Feature Extraction and Support Vector Machine. International Journal of Emerging Technology and Advanced Engineering (ISSN 2250-2459, Volume 2, Issue 11.

[5] W. Hsu, P.M.D.S Pallawala, Mong Li Lee, Kah-Guan Au Eong. 2001 The Role of Domain Knowledge in the Detection of Retinal Hard Exudates. Proc. IEEE Computer Society Conf. on Computer Vision and Pattern Recognition, 2, II-246 - II-251.

[6] R. Paisan, W. Nattapon, S. Pattanaporn, P. Ekchai, T. Montip. 2005 Screning for Diabetic Retinopathy in Rural Area Using Single-Field, Digital Fundus Images. Journal of Medical Association Thai, 88, 176-180.

[7] Nathan Silberman, Kristy Ahlrich, Rob Fergus and Lakshminarayanan Subramanian. 2010 Case for Automated Detection of Diabetic Retinopathy. Association for the Advancement of Artificial Intelligence.

[8] Jagadish Nayak, P Subbanna Bhat, Rajendra Acharya U, Lim Choo Min, Manjunath Gupta. 2007 Automated Identification of Different Stages of Diabetic Retinopathy using digital fundus images. Journal of Medical Systems, USA.

[9] Osareh, A.; Mirmehdi, M.; Thomas, B.; Markham, R. 2002 Classification and Localisation of Diabetic- Related Eye Disease. In Internat. European Conf. on Computer Vision, pp. 502-516.

[10] Wang; Hsu, H.; Goh, W.; Lee, K.G. 2000 An Effective Approach to Detect Lesions in Color Retinal Images. In Proceedings of IEEE Conf. on Computer Vision and Pattern Recognition, pp. 181-186.

[11] Phillips, R.P.; Forrester, J.; Sharp, P. 1993 Automated detection and quantification of retinal exudates. Graefe Arch Clin. Exp. Ophthalmol. 231, 90-94.

[12] Gardner, G.G.; Keating, D; Williamson, T.H.; Elliot, A.T. 1996 Automatic Detection of Diabetic Retinopathy using an Artificial Neural Network: a Screening Tool. Br. J. Ophthalmol. 80, 940-944.

[13] Dunn, J.C. 1973 A Fuzzy Relative of the ISODATA Process and Its Use in Detecting Compact WellSeperated Clusters. J. Cyber. 3, 32-57.

[14] Bezdek, J.C. 1981 Pattern Recognition with Fuzzy Objective Function Algorithms. Plenum: New York, NY, USA.

[15] Osareh, A.; Mirmehdi, M.; Thomas, B.; Markham, R. 2001 Automatic recognition of exudative maculopathy using fuzzy c-means clustering and neural networks. In Medical ImageUnderstanding Analysis; Claridge, E.; Bamber ,J., editors. BMVA Press, UK, pp. 49-52.

[16] Osareh, A.; Mirmehdi, M.; Thomas, B.; Markham, R. 2002 Comparative Exudate Classification using Support Vector Machines and Neural Networks. In Internat. Conf. on Medical Image Computingand ComputerAssisted Intervention, pp. 413-420.

[17] Vijaya Kumari V, SuriyaNarayanan N. 2010 Diabetic Retinopathy-Early Detection Using Image Processing Techniques. International Journal on Computer Science and Engineering 2(2): 357-361. 
[18] Berrichi Fatima Zohra, Benyettou Mohamed. 2009 Automated diagnosis of retinal images using the Support Vector Machine (SVM). Faculte des Science. Department of Informatique, USTO, Algerie.

[19] Neera Singh, Ramesh Chandra Tripathi. 2010 Automated Early Detection of Diabetic Retinopathy Using Image Analysis Techniques. International Journal of Computer Applications 8(2): 18-23.

[20] Jebarani Sargunar PN, Sukanesh R. 2009 Exudates Detection and Classification in Diabetic Retinopathy Images by Texture Segmentation Methods. International Journal of Recent Trends in Engineering 2(4): 148-150.

[21] Jonathan Goh, Lilian Tang, George Saleh, Lutfiah Al turk, Yu Fu, Antony Browne. 2009 Filtering Normal Retinal Images for Diabetic Retinopathy Screening Using Multiple Classifiers. International Conference on Information Technology and Applications in Biomedicine 1-4.

[22]

$$
\text { DIARETDB1 Database, }
$$
http://www2.it.lut.fi/project/imageret/diaretdb1/

[23]

DIARETDB0

Database, http://www2.it.lut.fi/project/imageret/diaretdb0/
[24] Musa, H.; Musa, Alci. 2005 Reliability analysis of microarray data using fuzzy c-means and normal mixture modeling based classification methods. Bioinformatics, $21,644-649$.

[25] Wang, X.Y.; Garibaldi, J.; Ozen, T. 2003 Application of The Fuzzy C-Means clustering Method on the Analysis of non Pre-processed FTIR Data for Cancer Diagnosis. In Internat. Conf. on Australian and New Zealand Intelligent Information Systems (ANZIIS), pp. 233-238.

[26] C. Jayakumari and T. Santhanam. 2007 Detection of Hard Exudates for Diabetic Retinopathy Using Contextual Clustering and Fuzzy Art Neural Network. Asian Journal of Information Technology 6(8): 842-846.

[27] Monireh Esnaashari, S. Amirhassan Monadjemi, and Gholamali Naderian. 2001 A Content-based Retinal Image Retrieval Method for Diabetes-Related Eye Diseases Diagnosis. International Journal of Research and Reviews in Computer Science (IJRRCS) Vol. 2, No. 6, ISSN: 2079-2557.

[28] Akara Sopharak, Matthew N. Dailey ,Bunyarit Uyyanonvara. 2010 Machine learning approach to automatic exudate detection in retinal images from diabetic patients. Journal of Modern Optics, 57(2):124135 